\title{
A Nucleolin-Targeted Multimodal Nanoparticle Imaging Probe for Tracking Cancer Cells Using an Aptamer
}

\author{
Do Won Hwang*1-3, Hae Young Ko*2-4 Jung Hwan Lee ${ }^{5}$, Hyungu Kang ${ }^{5}$, Sung Ho Ryu ${ }^{5}$, In Chan Song 6 , \\ Dong Soo Lee ${ }^{1,2,7}$, and Soonhag Kim ${ }^{8}$ \\ ${ }^{1}$ Programs in Neuroscience, Seoul National University, Seoul, Korea; ${ }^{2}$ Department of Nuclear Medicine, Seoul National University \\ College of Medicine, Seoul, Korea; ${ }^{3}$ Cancer Research Institute, Seoul National University College of Medicine, Seoul, Korea; \\ ${ }^{4}$ Institute of Radiation Medicine, Medical Research Center, Seoul, Korea; ${ }^{5}$ Aptamer Unit, Postech Biotech Center, Pohang University \\ of Science and Technology, Pohang, Kyungbuk, Korea; ${ }^{6}$ Department of Radiology, Seoul National University College of Medicine, \\ Seoul, Korea; ${ }^{7}$ Department of Nuclear Medicine and Department of Molecular Medicine and Biopharmaceutical Science, Seoul \\ National University College of Medicine, Seoul, Korea; and ${ }^{8}$ Laboratory of Molecular Imaging, CHA Stem Cell Institute, CHA \\ University, Seoul, South Korea
}

The recent advances in molecular imaging techniques, using cancer-targeting nanoparticle probes, provide noninvasive tracking information on cancer cells in living subjects. Here, we report a multimodal cancer-targeted imaging system capable of concurrent fluorescence imaging, radionuclide imaging, and $M R I$ in vivo. Methods: A cobalt-ferrite nanoparticle surrounded by fluorescent rhodamine (designated MF) within a silica shell matrix was synthesized with the AS1411 aptamer (MF-AS1411) that targets nucleolin (a cellular membrane protein highly expressed in cancer) using $N$-(3-dimethylaminopropyl)- $N$ ethylcarbodiimide (EDC). This purified MF-AS1411 particle was bound with 2-( $p$-isothio-cyanatobenzyl)-1,4,7-triazacyclonane1,4,7-triacetic acid ( $p$-SCN-bn-NOTA) chelating agent and further labeled with ${ }^{67} \mathrm{Ga}$-citrate (MFR-AS1411). The shape and size distribution of MFR-AS1411 were characterized by transmission electron microscope (TEM). The cellular distribution of the nucleolin protein using the MFR-AS1411 nanoparticle was detected by fluorescence confocal microscopy. Phantom MR images were obtained as the concentration of MFR-AS1411 increased, using a 1.5-T MRI scanner. In vivo ${ }^{67} \mathrm{G}$ a radionuclide imaging and MRI were performed using a $\gamma$-camera and a 1.5-T MR imager, respectively. Results: TEM imaging revealed MF and MFR-AS1411 to be spheric and well dispersed. The purified MFR-AS1411 nanoparticle showed specific fluorescence signals in nucleolin-expressing C6 cells, compared with MFR-AS1411 mutant (MFR-AS1411mt)-treated C6 cells. The rhodamine fluorescence intensity and ${ }^{67} \mathrm{Ga}$ activity of MFR-AS1411 were enhanced in a dose-dependent manner as the concentration of MFR-AS1411 was increased. The ${ }^{67} \mathrm{Ga}$ radionuclide was detected in both thighs of the mice injected with MFR-AS1411, whereas the MFR-AS1411 mutant (MFR-AS1411mt) administration revealed rapid clearance via the bloodstream, demonstrating that MFR-AS1411 specifically targeted cancer cells.

Received Aug. 27, 2009; revision accepted Oct. 7, 2009.

For correspondence or reprints contact: Soonhag Kim, Laboratory of Molecular Imaging, Department of Applied Bioscience, CHA Stem Cell Institute, CHA University, 605-21 Yoeksam 1-dong, Gangnam-gu, Seoul (135-081), Korea.

E-mail: kimsoonhag@empal.com

COPYRIGHT (c) 2010 by the Society of Nuclear Medicine, Inc.

${ }^{*}$ Contributed equally to this work.
Bioluminescence images in the C6 cells, stably expressing the luciferase gene, illustrated the in vivo distribution. T2-weighted MR images of the same mice injected with MFR-AS1411 showed dark T2 signals inside the tumor region, compared with the MRI signal of the tumor region injected with MFR-AS1411mt particles. Conclusion: We developed a nanoparticle-based cancerspecific imaging probe using the AS1411 aptamer in vivo and in vitro. This multimodal targeting imaging strategy, using a cancer-specific AS1411 aptamer, can be used as a versatile imaging tool for specific cancer diagnosis.

Key Words: multimodal image; cancer targeting; nanoparticles; aptamer; optical and radionuclide image

J Nucl Med 2010; 51:98-105

DOI: 10.2967/jnumed.109.069880

$\mathbf{T}$ he study of biologic imaging for cancer-specific targeting, using nanosized materials, has been an important part of theranostics use in drug development, nanosurgery, or medical nanosciences by visualization of target objects in animal subjects in vivo and at the molecular and cellular levels in vitro (1-4). Recently, a wide range of studies using multifunctional imaging probes has provided more precise imaging interpretation by overcoming the limitations of a single imaging modality. Several bi- or trimodal imaging probes have been devised and reported in the literature as successfully monitoring intracellular molecular activity and tracking stem cells in vivo (5-7).

Surface modifications of nanoparticles with antibodies, aptamers, peptides, or small molecules that bind to antigens present on the target cells or tissues have resulted in the development of sensitive and specific targeted imaging and diagnostic modalities for in vitro and in vivo applications $(8,9)$. Until now, monoclonal antibodies have been broadly used as ligands for molecular targeting because of their 
high affinity, specificity, and varied targeting availability. However, because of the long residence in the blood of antibodies, in vivo imaging using monoclonal antibodies as ligands deteriorates in quality over time. Therefore, we investigated the use of an aptamer for specific targeting of cancer cells, instead of an antibody using a multimodal nanoimaging probe.

Aptamers are synthetic DNA or RNA oligonucleotides that bind to target molecules with high affinity and specificity by a 3-dimensional structure. Because aptamers have low molecular weights, lack immunogenicity, and are readily available, these advantages make them good candidates for targeted cancer imaging and therapy. Recently, 2 reports on cancer cell imaging using the tenascin-C aptamer conjugated with a radioisotope and a prostate-specific membrane antigen aptamer conjugated with quantum dots showed specific targeting of cancer cells in vivo $(10,11)$.

In this study, we used a DNA aptamer (AS1411, also known as AGRO100) that binds to nucleolin in the plasma membrane (12). Nucleolin is highly expressed in continuously proliferating cells, such as cancer cells (13), and has a remarkable multifunction in the nucleus and cytoplasm and on the cell surface (14-16). The most representative function of AS1411 is its antiproliferative activity that blocks the antiapoptotic pathway by combining with nuclear factor- $\mathrm{kB}$ essential modulator in cancer cells (12).

The aim of this study was to develop an imaging system using a cancer-specific aptamer, by examining the in vivo distribution of a multimodal nanoparticle imaging probe in living subjects. An AS1411-laden nanoparticle imaging probe was constructed using a magnetic fluorescence nanoparticle for the investigation of an aptamer-based multimodal cancer imaging probe, using AS1411 aptamers that bind to nucleolin proteins in the cellular membrane of cancer cells. Here, we report an experimental investigation of this multimodal imaging system with fluorescence imaging, radionuclide imaging, and MRI modalities in living animals.

\section{MATERIALS AND METHODS}

\section{Construction of MF-AS1411 Particle}

$\mathrm{MNP} @ \mathrm{SiO}_{2}$ (RITC)-(PEG)/COOH/pro-N/NH 2 nanoparticles (MF, $2 \mathrm{mg} / \mathrm{mL}$ ) were purchased from Biterials (Seoul, Korea) and prepared as previously described (17). Carboxyl moieties $(1.1 \times$ $10^{4} /$ nanoparticle) of the MF particles (size, $\sim 50 \mathrm{~nm}$; hydrodynamic diameter, $58.1 \mathrm{~nm}$ ) were covalently linked to a $5^{\prime}-\mathrm{NH}_{2}-$ modified AS1411 aptamer (5'-TTGGTGGTGGTGGTTGTGGTG GTGGTGG-3') using $N$-(3-dimethylaminopropyl)- $N$-ethylcarbodiimide (EDC) (MF:aptamer molar ratio in conjugation reaction, 1:3) (Sigma) for $1 \mathrm{~h}$ at room temperature (18). The MF-AS1411 conjugates were washed off by centrifugation at $22,250 \mathrm{~g}$ for 10 min and resuspended in selection buffer solution $(50 \mathrm{mM}$ Tris$\mathrm{HCl}, \mathrm{pH} 7.4)$. Amine groups $\left(6.4 \times 10^{4} /\right.$ nanoparticle $)$ protected by the Fmoc group were released by $20 \%$ piperidine (Sigma) in an $\mathrm{N}, \mathrm{N}$-dimethylformamide solution (Sigma). After $1 \mathrm{~h}$ of incubation, the MF-AS1411 particles were washed off twice with Tris buffer (pH 7.4) and briefly sonicated. To confirm the conjugation pattern of MF-AS1411, the conjugated MF-AS1411 and MF particle were loaded onto each well of $0.5 \%$ agarose gel with electrophoresis buffer $(0.5 \times$ Tris-acetate-ethylenediaminetetraacetic acid) and run at $100 \mathrm{~V}$.

\section{Transmission Electron Microscope (TEM) Analysis}

Negatively stained specimens were prepared on a formvarcoated grid, and the AS1411 aptamer was stained with $2 \%$ aqueous uranyl acetate. TEM study was performed using a JEM 1010 (JEOL) at $80 \mathrm{kV} .{ }^{67} \mathrm{Ga}$-labeled MF-AS1411 particles (MFRAS1411) $(n=3)$ were fixed using $2 \%$ formaldehyde for $10 \mathrm{~min}$. MF particle was also prepared as a control $(n=3)$ by being fixed using $2 \%$ formaldehyde. Electron microscope digital images were recorded using a Gatan cooled charge-coupled device camera.

\section{Cell Cullture}

C6 rat glioma cells were cultured in Dulbecco's modified Eagle's medium (Invitrogen), supplemented with $10 \%$ heat-inactivated $\left(65^{\circ} \mathrm{C}\right.$ for $\left.20 \mathrm{~min}\right)$ fetal bovine serum (Invitrogen) with $1 \%$ antibiotics (Invitrogen), in a standard incubator $\left(5 \% \mathrm{CO}_{2}\right.$ atmosphere at $37^{\circ} \mathrm{C}$ ). The cells were split at regular intervals. C6 stably expressing luciferase gene regulated by cytomegalovirus promoter was generated by the selection with several concentrations of geneticin (Invitrogen) over $2 \mathrm{wk}$.

\section{Confocal Microscopy of MF-AS1411 Targeted to $\mathrm{C} 6$ Cells}

C6 cells $\left(1 \times 10^{5}\right.$ cells/well $)$ were seeded on 25 -mm-diameter glass cover slips, and the cells were grown for $24 \mathrm{~h}$ at $37^{\circ} \mathrm{C}$. Before treatment with MF-AS1411 conjugates, the cells were incubated for $30 \mathrm{~min}$ at $4^{\circ} \mathrm{C}$ and then washed with phosphate-buffered saline (PBS). The conjugated particles were added to cells under Tris buffer ( $\mathrm{pH} 7$ ). After incubation for $30 \mathrm{~min}$ at $4^{\circ} \mathrm{C}$, the cells were fixed by gently shaking for 20 min with $4 \%$ formaldehyde solution (Sigma). Cells were then washed 3 times with PBS for $10 \mathrm{~min}$ and cover-slipped with mounting medium containing 4',6-diamidino-2phenylindole dihydrochloride solution (Vector Laboratories, Inc.). The images were acquired with confocal laser scanning microscopy (LSM 510; Carl Zeiss) with an excitation wavelength of $556 \mathrm{~nm}$ and an emission wavelength of $570 \mathrm{~nm}$.

In Vitro Fluorescence Analysis and Stability Testing of Rhodamine Fluorescence in MFR-AS1411 Particle

Each concentration $(3,6,9,12,15$, and 18 pmol) of MF particle was first conjugated with the AS1411 aptamer at a 1:3 ratio of MF to AS1411 aptamer. The MF-AS1411 nanoparticles were added to the C6 cells and incubated for $1 \mathrm{~h}$. The cells treated with the MFAS1411 particle were harvested by trypsinization after rinsing with PBS. The collected cells were transferred into dark 96-well microplates, and their fluorescence intensities were measured using the Infinite M200 (Tecan, GmbH, SZ). For fluorescence stability testing, $5 \mathrm{pmol}$ of MF particles were conjugated with 15 pmol of the AS1411 aptamer using EDC. After the MF-AS1411 was synthesized with 2-( $p$-isothio-cyanatobenzyl)-1,4,7-triazacyclonane-1,4,7-triacetic acid ( $p$-SCN-bn-NOTA), the same amount of the conjugated MFR-AS1411 particle was added to Eppendorf tubes containing PBS solution and measured using the fluorescence imager (Tecan, GmbH, SZ).

\section{Radiolabeling of MF-AS1411 with ${ }^{67} \mathrm{Ga}-$ Citrate and In Vitro Experiment with MFR-AS1411 \\ To react the MF-AS1411 particle with $p$-SCN-Bn-NOTA (500 $\mathrm{gmol}^{-1}$; concentration, $5 \mathrm{mg} / \mathrm{mL}$ ), the amino moieties of MF-}


AS1411 were synthesized using a $p$-SCN-Bn-NOTA adduct with $\mathrm{NaHCO}_{3}$ buffer solution, at a MF-to- $p$-SCN-Bn-NOTA reaction ratio of $1: 5$ under agitation at $4^{\circ} \mathrm{C}$ overnight (19). MF-AS1411 synthesized with $p$-SCN-Bn-NOTA was labeled with ${ }^{67} \mathrm{Ga}$-citrate $\left(37 \mathrm{MBq}[1 \mathrm{mCi}] / 400 \mu \mathrm{L}\right.$ ) under $0.2 \mathrm{M} \mathrm{Na} \mathrm{HPO}_{4} / \mathrm{NaH}_{2} \mathrm{PO}_{4}$ (Sigma) buffer ( $\mathrm{pH}$ 6.5) and incubated for $1 \mathrm{~h}$. The particles of MF-AS1411-NOTA labeled with ${ }^{67} \mathrm{Ga}$-citrate (MFR-AS1411) were checked by thin-layer chromatography (TLC) to analyze the labeling efficiency. The reaction mixture of radiolabeled MFAS1411 was applied to instant thin-layer chromatography-silica gel (Pall, Inc.) and separated in a simple beaker containing $0.1 \mathrm{M}$ citric acid solvent. The radioactivity was measured using an AR2000 TLC imaging scanner (Bioscan, Inc.). The labeling efficiency for the radioactivity bound to the MF-AS1411 particles was measured as percentage of ROIs on TLC paper silica gel.

To examine the specific targeting of MFR-AS1411 nanoparticle to the $\mathrm{C} 6$ cells by measuring radioactivity of ${ }^{67} \mathrm{Ga}$-citrate in MFRAS1411, the synthesized MF-AS1411-NOTA particles at the different concentrations (MF particle, 3, 6, 9, 12, 15, and 18 pmol) were labeled with ${ }^{67} \mathrm{Ga}$-citrate $(37 \mathrm{MBq}$ for $18 \mathrm{pmol}$ of $\mathrm{MF}$ concentration) through a serial dilution method. Radioactivity of ${ }^{67} \mathrm{Ga}$-citrate was measured for $1 \mathrm{~min}$ at each group using a $\mathrm{NaI}$ well counter (Cobra II; Canberra Packard).

\section{In Vitro Phantom MRI Analysis}

Phantom MRI studies were performed using mixtures of MFRAS1411 at 6 different concentrations $(0.2,0.4,0.8,1.6,3.2$, and $6.4 \mathrm{mM})$. Six samples in a variety of concentrations of MFRAS1411mt were also prepared. One day after $5 \times 10^{5}$ C6 cells were seeded into 24-well plates, each MFR-AS1411 sample was added to the $\mathrm{C} 6$ cells and incubated for $1 \mathrm{~h}$. The collected cells were transferred into Eppendorf tubes after several washing steps with PBS solution. The T2 MRI signal intensities of phantom experiments, for the different concentrations of the MFR-AS1411 particles, were measured using $1.5 \mathrm{~T}$ on a whole-body MRI scanner (GE Healthcare).

\section{Scintigraphic Imaging of MFR-AS1411 Particle in Tumor-Bearing Nude Mice}

The C6 cells $\left(1 \times 10^{6}\right)$ stably expressing the luciferase gene were harvested in PBS solution, for in vivo cellular tracking of C6 cells, and injected into the left and right thighs of nude mice. The subcutaneously implanted C6 cells were grown until a tumor size of approximately 1-2 cm was reached (observed maximum tumor size, $1.6 \mathrm{~cm}$ ). MFR-AS1411 was separated from free ${ }^{67} \mathrm{GaCl}_{3}$ by centrifugation at $22,250 \mathrm{~g}$ for $10 \mathrm{~min}$, was thoroughly washed in Tris buffer, and underwent a brief sonication. Two weeks after the implantation of C6-expressing luciferase, MFR-AS1411 was systemically administered into C6-bearing nude mice. For the in vivo negative control group, MFR-AS1411mt (AS1411 mutant form for which the core nucleotides $\mathrm{G}$ in AS1411 aptamer were substituted with $C$ ) was injected into other nude mice via the tail vein. BALB/c nude mice (10 wk old; $n=3$ ) were used for MFRAS1411-treated mice and the MFR-AS1411mt (control) group, respectively. All mice received intraperitoneal injections of $50 \mu \mathrm{L}$ of a ketamine and xylazine (2:1) solution for anesthesia. For the acquisition of ${ }^{67} \mathrm{Ga}$ scintigraphic images, the mice injected with MFR-AS1411 were placed in spread-prone position, and wholebody scintigraphic images were acquired using a $\gamma$-scintillation camera (ON 410; Ohio Nuclear) equipped with a pinhole collimator. The accumulation of ${ }^{67} \mathrm{Ga}$ radioactivity was examined in each thigh of the mice, with the acquisition time for $10 \mathrm{~min}$. For the in vivo cellular tracking of C6 cells, bioluminescence images were acquired after $3 \mathrm{mg} / 0.1 \mathrm{~mL}$ of luciferin were intraperitoneally injected. Animals were positioned in an IVIS-200 equipped with a cooled charge-coupled device camera (Xenogen), and bioluminescence images were acquired for $3 \mathrm{~min}$. ROIs were drawn around the area of uptake in the right and left thighs on the $\gamma$-camera images. The average counts per pixel were recorded for both thighs of the mouse. All experimental animals were housed under specific pathogen-free conditions and handled in accordance with the guidelines issued by the Institutional Animal Care and Use Committee of Seoul National University Hospital.

\section{Acquisition of MR Images in Nude Mice}

To determine whether the transplanted cells were visible by MRI, as soon as scintigraphic images for the 24-h group were acquired in mice, then the T2*-weighted images were obtained in the same mice using a fast low-angle shot sequence. During MRI experiments, the animals were sedated with $2 \%$ isoflurane in a mixture of $\mathrm{O}_{2}$ and $\mathrm{N}_{2} \mathrm{O}$ at a flow rate of $1 \mathrm{~L} / \mathrm{min}$ through the nose cone. The temperature and respirations were monitored by a rectal thermistor and pillow. Mice were positioned in an animal coil box $(n=3$, mice $)$, and in vivo MR images were acquired using a $1.5-\mathrm{T}$ MR imager (GE Healthcare).

\section{Statistical Analysis}

Data are represented as means \pm SEM and were calculated using the Student $t$ test. Statistical significance was accepted at $P$ values of less than 0.05 .

\section{RESULTS}

\section{Design of Probe and In Vitro Evaluation of AS1411 Aptamer Specificity}

To construct the cancer-targeting multimodal imaging probe, ${ }^{67} \mathrm{Ga}-\mathrm{MNP} @ \mathrm{SiO}_{2}$ (RITC)-PEG/NH $2-\mathrm{AS} 1411$ (MFRAS1411) was designed (Fig. 1). The MFR-AS1411 nanoparticle was composed of magnetic cobalt ferrite in the central core and rhodamine B isothiocyanate fluorescence dye $(\mathrm{MF})$ coated with a silica shell. In addition, polyethylene glycol (PEG) $\left(1 \times 10^{4} /\right.$ nanoparticle $)$, Fmoc-protected amine moieties, and a carboxyl group were surrounded with the surface of particles, which were further labeled with the AS1411 aptamer and p-SCN-bn-NOTA chelator (Fig. 1). TEM imaging of the MF particles showed a homogeneous distribution of the magnetic cobalt-ferrite coated with rhodamine fluorescence dye (Fig. 2A; hydrodynamic particle size, $58.1 \mathrm{~nm}$ ). After the removal of the Fmoc-protection group from the MF-AS1411 particles, the MF-AS1411 particles were labeled with ${ }^{67} \mathrm{Ga}$-citrate after the introduction of the NOTA chelating agents (MFRAS1411). TEM images of the MFR-AS1411 particles also showed that the AS1411 aptamer in the MFR-AS1411 was detected as a dark frame line on $2 \%$ uranyl acetate-filled specimens and measured about $68.1 \mathrm{~nm}$ (hydrodynamic size) (Fig. 2A). In addition, when the MFR-AS1411 particles were incubated with PBS, in a time-dependent manner $(0,1$, 3,6 , and $24 \mathrm{~h}$ ) to measure the stability of the rhodamine dye in the MFR-AS1411, the fluorescence intensity of MFR-AS1411 was maintained up to $24 \mathrm{~h}$ (Supplemental 


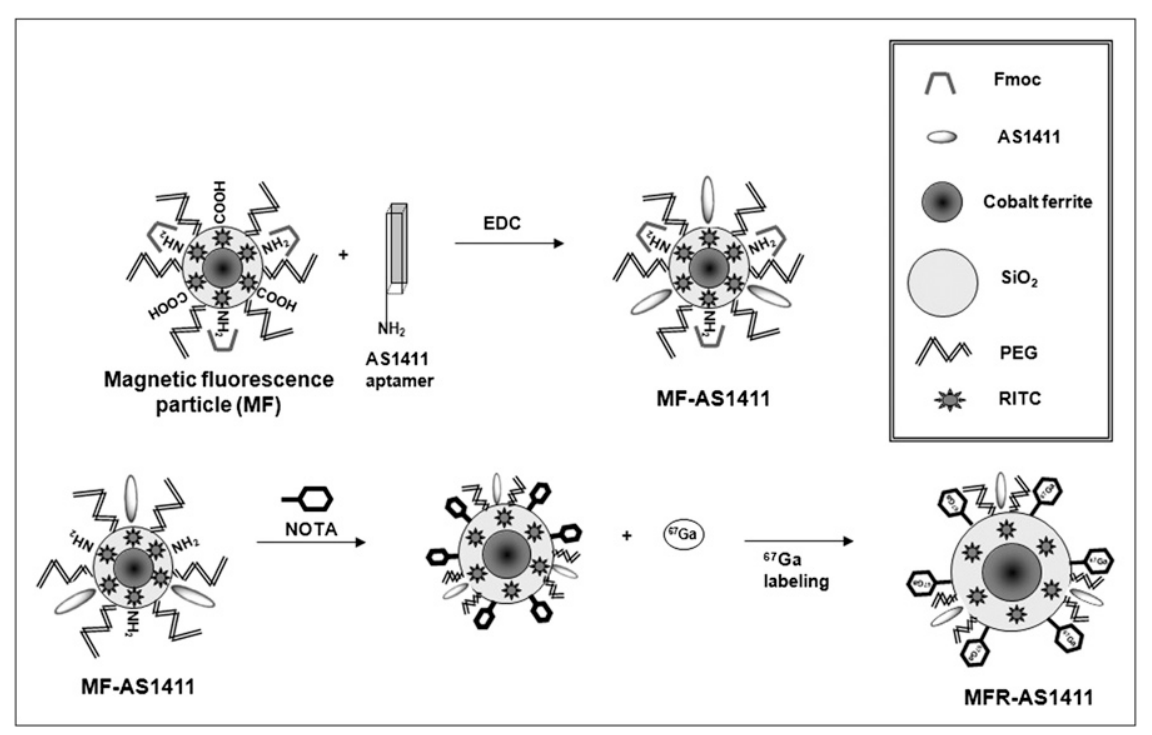

FIGURE 1. Schematic illustration of steps involved in preparation of MFRAS1411 and in vitro fluorescence cancer targeting measured by confocal microscopy. MF particles had carboxyl group and Fmoc-protected amine moiety, which was coupled with amineterminated AS1411 aptamer using EDC (MF-AS1411). After reaction of MFAS1411 with $p$-SCN-bn-NOTA, particles were reacted with ${ }^{67} \mathrm{Ga}$-citrate to form MFR-AS1411.

Fig. 1A; supplemental materials are available online only at http://jnm.snmjournals.org). Gel electrophoresis results revealed the different size of the free MF particle, compared with the MF-AS1411 group, demonstrating the efficient conjugation between the MF particles and the AS1411

aptamer (Supplemental Fig. 1B). To examine the cancertargeting specificity of the AS1411 aptamer, MF-AS1411 or MF-AS1411mt nanoparticles were added to the C6 cells. Fluorescence confocal microscopy showed greater rhodamine fluorescence in the MF-AS1411-treated C6 cells than

A

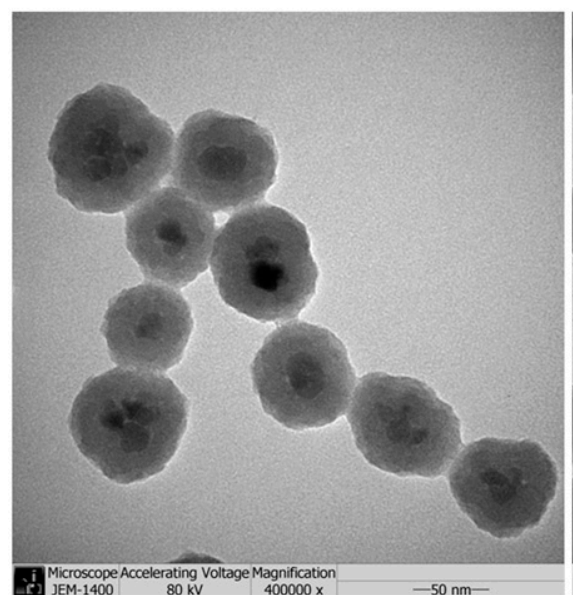

B

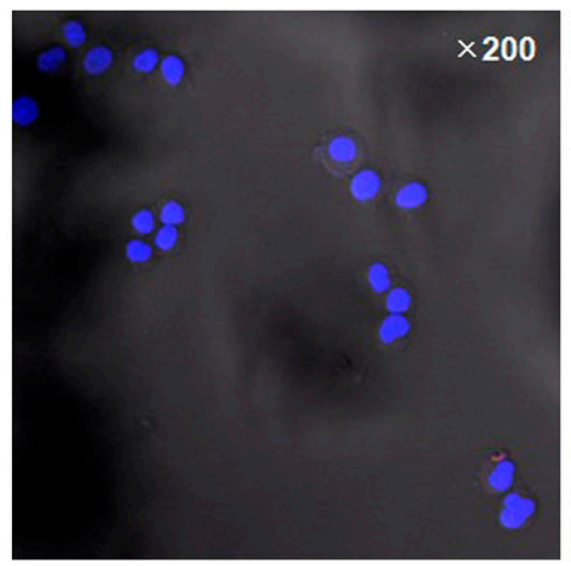

MFR-AS1411

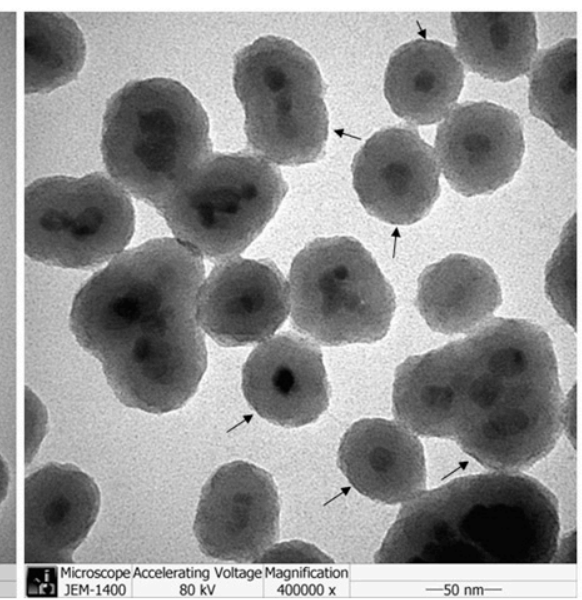

MF-AS1411

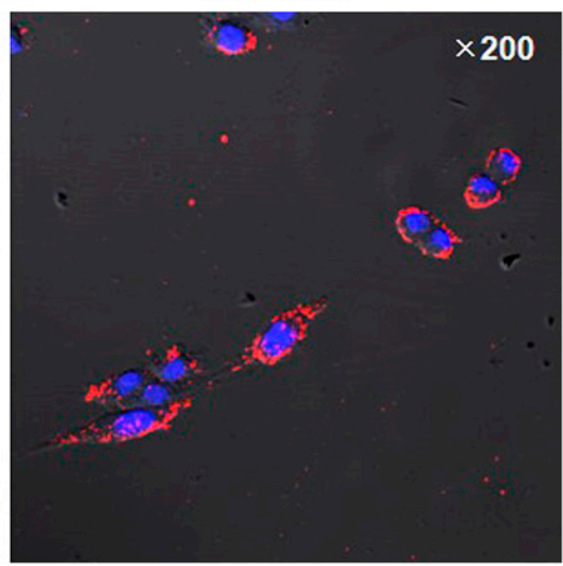

FIGURE 2. (A) TEM images were obtained in MF particles consisting of magnetic cobalt ferrite core, rhodamine dye, and MFR-AS1411, which was MFlabeled with AS1411 aptamer and ${ }^{67} \mathrm{Ga}$ citrate. Uranyl acetate (2\%) was used to stain AS1411 aptamer. Dark staining of AS1411 in MFR-AS1411 was detected on surface of MFR-AS1411 particles (uranyl acetate-stained AS1411, black arrow). Scale bar $=50 \mathrm{~nm} .{ }^{*} P<0.005$. (B) After MF nanoparticles were conjugated with AS1411 aptamer using EDC, MF-AS1411 conjugates were treated into C6 cells. MF-AS1411 specifically targeted C6 glioma cells, as determined by laser scanning confocal microscopy (red, MF-AS1411; blue, 4',6-diamidino2-phenylindole dihydrochloride). No fluorescence signal was detected in MF-AS1411mt-treated group. 
in the MF-AS1411mt-treated C6 cells (Fig. 2B). In vitro fluorescence microscopy revealed detailed information on the location of nucleolin in cells using the AS1411 aptamer, with good resolution in the C6 cells (Fig. 2B).

\section{Multimodal Probe Activity of MFR-AS1411 Nanoparticle in C6 Cells}

To quantify the optical specificity for targeting cancer cells using MF-AS1411 or MF-AS1411mt, serially increased concentrations of MF-AS1411 were added to the C6 cells. The results showed a dose-dependent and significant increase in the fluorescence signal of the MF-AS1411-treated C6 cells, compared with the MF-AS1411mt-treated C6 cells (Fig. 3A).

The ${ }^{67}$ Ga-labeling efficiency of MFR-AS1411 was measured using quantitative TLC analysis. The results showed that approximately $40 \%$ of the MFR-AS1411 incorporated ${ }^{67}$ Ga-citrate (Supplemental Fig. 2A). The binding stability between $p$-SCN-bn-NOTA and ${ }^{67} \mathrm{Ga}$-citrate, measured by TLC analysis over time, was relatively stable up to $12 \mathrm{~h}$ in mouse serum and PBS solution (data not shown).

After the MF-AS1411 particles labeled with ${ }^{67} \mathrm{Ga}$-citrate using NOTA chelator (MFR-AS1411) at various concentrations (MF particle, 3, 6, 9, 12, 15, and 18 pmol) were incubated with the $\mathrm{C} 6$ cells for $1 \mathrm{~h},{ }^{67} \mathrm{Ga}$ radioactivity of the collected C6 cells was gradually increased depending on the concentration of the MFR-AS1411 particle, showing higher radioactivity than the MFR-AS1411mt-treated group (Fig. 3B). The radioactivity of MFR-AS1411-treated C6 cells was higher than that of MFR-AS1411mt-treated C6 cells at each concentration point. For the in vitro assay of MFR-AS1411 in the cells, the synthesized MF-AS1411-
NOTA particles at the different concentrations were labeled with ${ }^{67} \mathrm{Ga}$-citrate $(37 \mathrm{MBq}$ for $18 \mathrm{pmol}$ of MF concentration) through a serial dilution method. ${ }^{67} \mathrm{Ga}$-citrate reaction activities were measured using a NaI well counter. Phantom MR images were obtained at 6 different concentrations $(0.2,0.4,0.8,1.6,3.2$, and $6.4 \mathrm{mM})$ of the MFR-AS1411 nanoparticle. The same concentrations of MFR-AS1411mt were prepared as 6 samples. The prepared MFR-AS1411 (or MFR-AS1411mt) particle was incubated with the C6 cells for $1 \mathrm{~h}$. The T2-weighted MR images showed a gradual decrease in the magnetic signal intensity as the concentration of MFR-AS1411 increased. The specific relaxivity was $9.04 \mathrm{mM}^{-1} \mathrm{~s}^{-1}$ (Fig. 3C). The magnetic signals of the MFR-AS1411 particles were also gradually decreased as the concentration of MFR-AS1411 increased, showing a specific relaxivity of $82.1 \mathrm{mM}^{-1} \mathrm{~s}^{-1}$ (Supplemental Fig. 2B).

\section{In Vivo Radionuclide Detection and MRI of Systemic MFR-AS1411}

Twenty-four hours after the purified MFR-AS1411 particles were systemically administered into the nude mice bearing C6 tumors, ${ }^{67} \mathrm{Ga}$ radioactivity was detected in the MFR-AS1411-administered mice at the site of the administration; the MFR-AS1411mt-administered mice showed rapid clearance through the bloodstream, indicating that the MFR-AS1411 specifically targeted the tumors (Fig. 4A). For the in vivo cellular tracking of C6 cells, C6 cells that express constant firefly luciferase were used as an internal control for the in vivo normalization of the radioactivity intensities. The bioluminescence images of C6 cells stably expressing luciferase in both thighs of MFR-AS1411- or MFRAS1411mt-injected mice were clearly visualized, whereas
FIGURE 3. In vitro cancer targeting using different imaging modalities. (A) C6 cells were treated with diluted concentration of MF-AS1411 (or MFAS1411mt). Gradual increase of fluorescence intensity in MF-AS1411treated group was observed, compared with MF-AS1411mt group. (B) After synthesizing MF-AS1411 (or MFAS1411mt) using $p$-SCN-bn-NOTA and incubating overnight, MF-AS1411 and MF-AS1411mt were labeled with ${ }^{67} \mathrm{Ga}$ citrate in buffer solution for $1 \mathrm{~h}$. Concentration of MFR-AS1411 particles was critically dependent on ${ }^{67} \mathrm{Ga}$ radioactivity in $\mathrm{C} 6$ cells. ${ }^{\star} P<0.05 .{ }^{* *} P<$ 0.005. (C) Phantom studies were performed at various concentrations of MFR-AS1411 (or MFR-AS1411mt) mixture. Gradual decrease of MRI signals in MFR-AS1411-treated group were detected, compared with MFR-AS1411mttreated group, on 1.5-T MRI system.
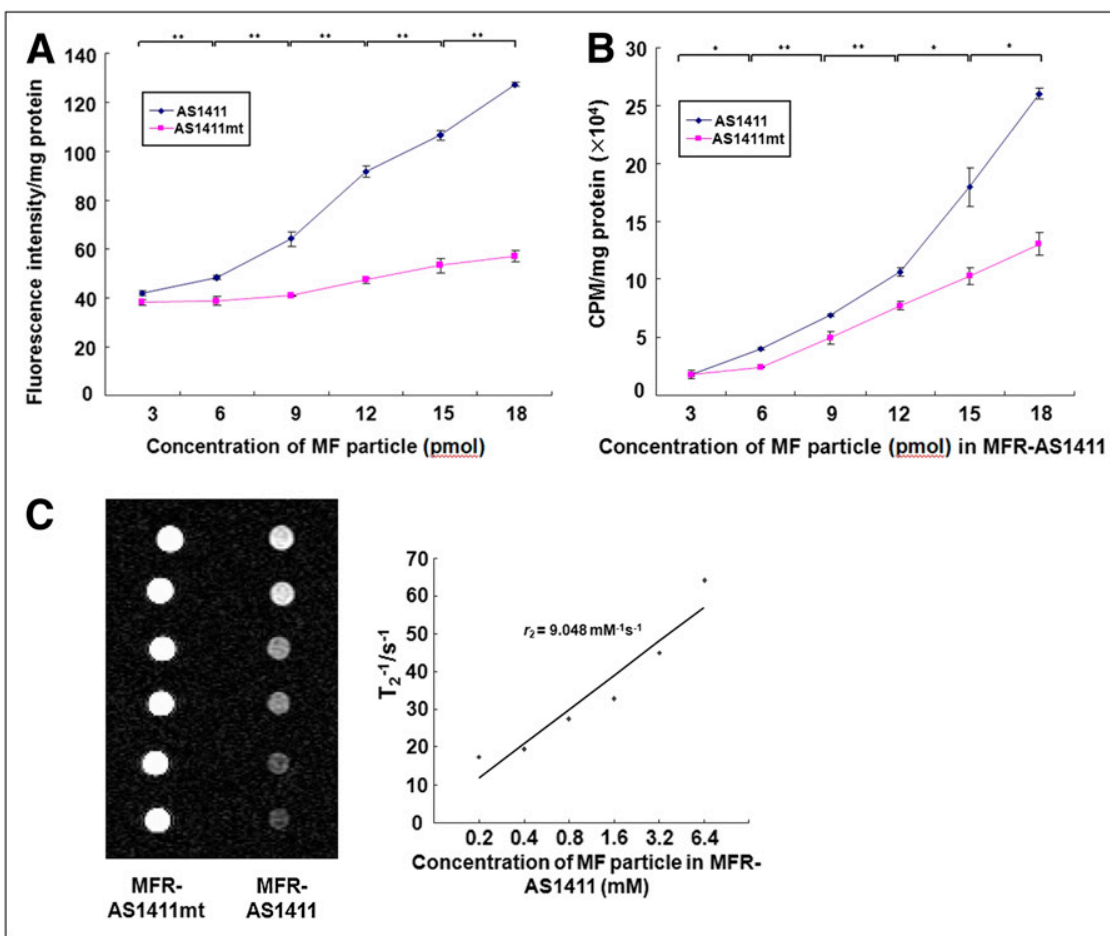


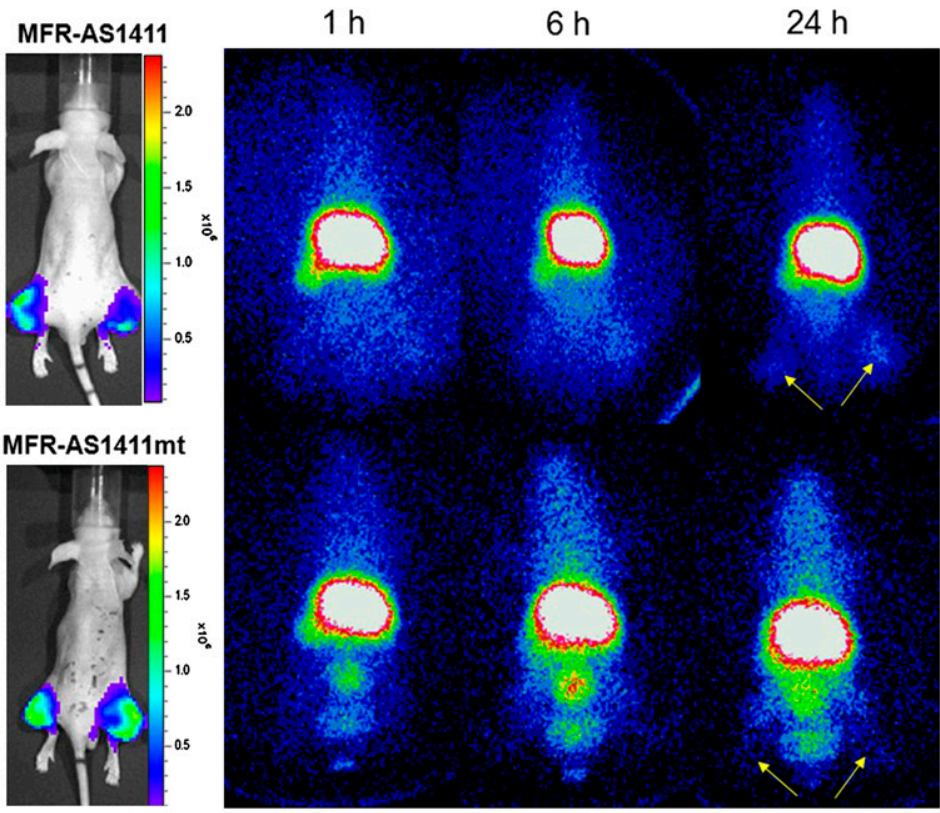

B
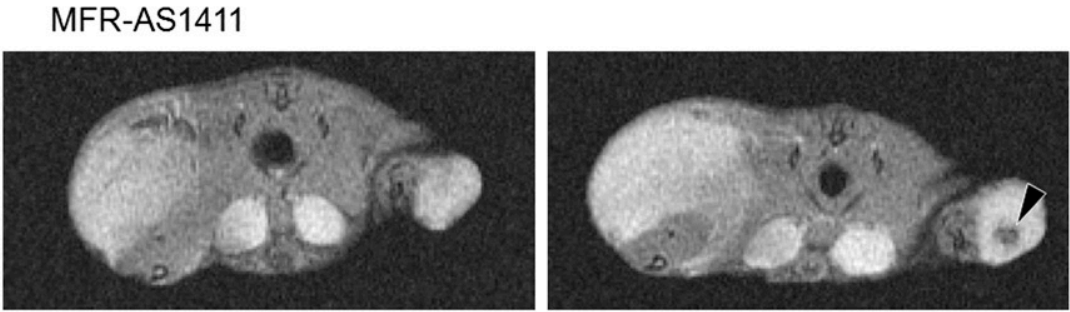

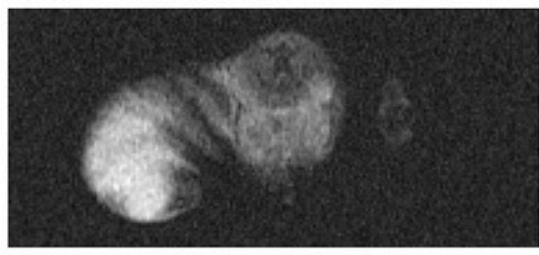

Before injection

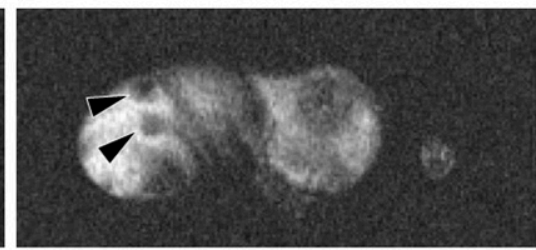

After $24 \mathrm{~h}$

C

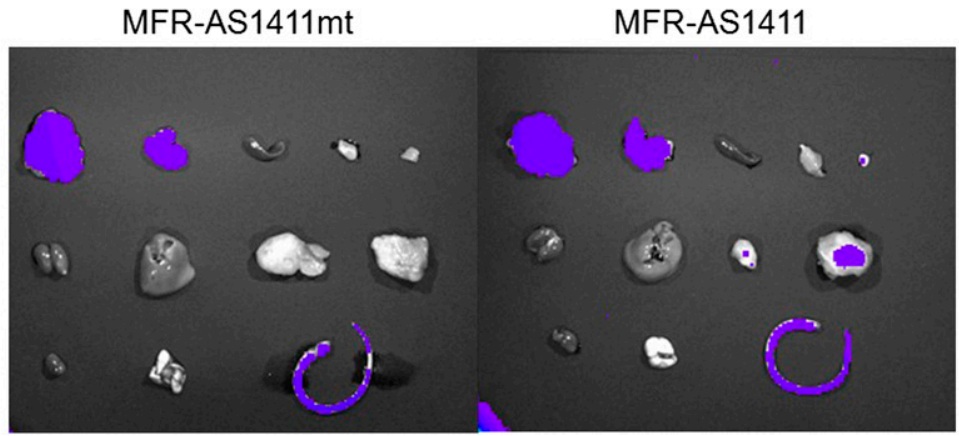

FIGURE 4. In vivo multimodal cancer targeting and imaging using MFRAS1411 particles. (A) MFR-AS1411 particles were intravenously injected into tumor-bearing mice, and radionuclide images were acquired at 1,6 , and $24 \mathrm{~h}$ after injection. Scintigraphic images of C6 tumors in mice that received MFRAS1411 showed that C6 tumors had accumulated MFR-AS1411 at $24 \mathrm{~h}$ after injection but did not accumulate MFRAS1411mt $(n=3)$. Tumor growth patterns were followed using bioluminescence signals acquired from luciferase-expressing $\mathrm{C} 6$ cells. (B) MR images of tumor-bearing mice before and after injection of MFR-AS1411 were acquired. Dark signal intensities at tumor sites were detected in MFR-AS1411-injected mice (arrowhead). (C) Tumors were isolated and their fluorescence verified using IVIS200 system. Fluorescence signal at tumor site injected with MFRAS1411 was detected, compared with tumors injected with MFR-AS1411mt. Isolated organs in order from upper left to lower right were intestine, liver, spleen, muscle, fat, kidney, stomach, right tumor, left tumor, heart, lung, and tail. the radionuclide signal was found in both thighs only of the MFR-AS1411-injected mouse (Fig. 4A). Quantitative ROIs showed higher radioactivity in the MFR-AS1411-injected group than in MFR-AS1411mt group (Supplemental Fig. $3 \mathrm{~A}, n=3$ ). As shown in Figure 4A, the specific cancer targeting of MFR-AS1411 nanoparticle using the ${ }^{67} \mathrm{Ga}$ radioisotope was detected $24 \mathrm{~h}$ after systemic injection of MFR-AS1411. However, the systemic injection of MFR-
AS1411 nanoparticle revealed intense nonspecific liver accumulation by the mononuclear phagocytic system.

MR images of the same mice injected with the MFRAS1411 were obtained before and $24 \mathrm{~h}$ after injection (intravenously). T2-weighted MR images from tumorbearing mice injected with MFR-AS1411 showed the MFR-AS1411 particles as black spots (Fig. 4B). No T2negative images were observed in the MFR-AS1411mt- 
injected tumor-bearing mice (Supplemental Fig. 3B). To confirm whether the multimodal cancer-targeting images were acquired by MFR-AS1411 particles, the fluorescence activity of several organs and tumor masses was evaluated. High fluorescence signals were obtained from the intestine, liver, and tumors, demonstrating the tumor-specific accumulation of MFR-AS1411 (Fig. 4C).

\section{DISCUSSION}

Multimodal imaging platforms including triple-fusion reporter gene imaging and dual-imaging nanoparticles have recently been developed $(20,21)$. The 2 major advantages of integrated multimodality imaging systems are that they offer the benefits of complementary modalities that tend to eliminate the shortcomings of individual imaging modalities and that the described multifunctional system offers a broad range of imaging possibilities, ranging from in vitro cellular studies using fluorescence materials to bioluminescence imaging in animal models and radionuclide and MRI for potential diagnostic and therapeutic human application (22).

In this study, in vitro fluorescence microscope images revealed detailed information on the cellular location of nucleolin with the AS1411 aptamer, showing good resolution at the single-cell level (Fig. 2B). However, as for the in vivo fluorescence image, the emitted fluorescence signals can be attenuated in deep-tissue areas. In this case, radionuclide imaging might be preferred for the acquisition of in vivo images over time, providing high sensitivity regardless of tissue depth. We chose the ${ }^{67} \mathrm{Ga}$ radioisotope for cancer-targeting radionuclide imaging because of its characteristics, including the appropriate half-life (78.2 h), to enable longer image tracking. As shown in Figure 4A, the specific cancer targeting of MFR-AS1411 nanoparticles using the ${ }^{67} \mathrm{Ga}$ radioisotope was detected $24 \mathrm{~h}$ after systemic administration of the MFR-AS1411. However, the systemic injection of the MFR-AS1411 nanoparticle revealed intense nonspecific liver accumulation by the mononuclear phagocytic system. Although the surface of the MFR-AS1411 particles contains PEG moieties to reduce nonspecific uptake of nanoparticles by the mononuclear phagocytic system, the size of the MFR-AS1411 allowed natural accumulation to occur in the liver (Fig. 4A). Also, in Figure 4C we detected nonspecific fluorescence signals from the MFR-AS1411 in the tail vein. To prevent MFR-AS1411 from being retained for long periods in the tail vein, it was necessary to administer a physiologic buffer, such as PBS, as soon as the MFR-AS1411 particles were injected into the tail vein.

Compared with the PET radioisotopes (which have a short half-life), several radioisotopes emitting $\gamma$-rays, such as ${ }^{111}$ In (half-life, $\sim 2.8 \mathrm{~d}$ ) and ${ }^{67} \mathrm{Ga}$, have an acceptable halflife for tracking cancers using nanoparticle-based aptamer systems and could provide long-term image tracking.

A recent study developing an in vivo tumor-targeting system using a tumor-specific aptamer showed a significant accumulation of radiolabeled aptamer into the tumor target site, followed by rapid clearance of the aptamer from the advantage of the small size of the aptamer (10). Compared with this system, the subsequent studies including the nonspecific uptake of the developed imaging probe to the liver are necessary to improve the optimal biodistribution using nanoparticle-based imaging probes. A potential solution to improve the biodistribution, such as the study to avoid natural accumulation as nonspecific uptake into the liver by changing a particle size, will be the next step in the development of this multimodal diagnostic probe for cancer imaging.

In order that nanoparticles are applied for clinical study, it is important to note that cellular toxicity of nanoparticles reveals critical limitations for human use. From a variety of toxicity studies that measured body weight and chromosomal aberrations, Kim et al. (23) reported that MF nanoparticles did not cause apparent cellular toxicity in vivo or in vitro. Therefore, MF nanoparticles, as used in this study, in which the magnetic core of the MF nanoparticle is surrounded by a stable silica shell, might be acceptable for clinical studies in humans.

In the present study, the MFR particles consistently allowed for fluorescence imaging, radionuclide imaging, and MRI in vitro and in vivo. These findings suggest that MFR-AS1411 nanoparticles are useful for the multimodal imaging of disease, theranostics (including cancer and neuronal diseases), and the study of cellular metabolism. In addition, this cancer-targeting multimodal nanoparticle probe based on aptamers could provide accurate cancer detection through integrated information acquired from in vitro fluorescence imaging, in vivo radionuclide imaging, and MRI signals.

\section{CONCLUSION}

We developed a cancer-targeted imaging probe, using a multimodal nanoparticle conjugated with the AS1411 aptamer, which targets nucleolin protein highly expressed on the membrane of cancer cells. Our MFR-AS1411 nanoparticle successfully targeted cancer cells and was monitored by fluorescent, radioisotope, and MRI modalities in vivo and in vitro. This multimodal cancer-targeted imaging strategy using the AS1411 aptamer-labeled MFR particles provides a versatile imaging tool that can enhance the diagnosis and treatment of cancer patients. Multifunctional imaging modality platforms based on a combination of nanotechnology and molecular imaging represent the leading edge of imaging research and development and will undoubtedly broaden our understanding of molecular diagnostics and therapeutics in the near future.

\section{ACKNOWLEDGMENTS}

This work was supported by the Brain Research Center of the 21st Century Frontier Research Program funded by the Ministry of Science and Technology (2009K001257), 
the WCU project of the MEST and the NRF (R31-2008-00010103-0), the National R\&D Program for Cancer Control of Ministry of Health and Welfare (0820320), the National Research Foundation of Korea (no. 20090084640), and a grant of the Korea Healthcare Technology R\&D Project, Ministry for Health, Welfare \& Family Affairs, Republic of Korea (A085136).

\section{REFERENCES}

1. Leary SP, Liu CY, Yu C, Apuzzo ML. Toward the emergence of nanoneurosurgery: part II-nanomedicine: diagnostics and imaging at the nanoscale level. Neurosurgery. 2006;58:805-823.

2. Suri SS, Fenniri H, Singh BL. Nanotechnology-based drug delivery systems. $J$ Occup Med Toxicol. 2007;2:16.

3. Liu Y, Miyoshi H. Nakamura M. Nanomedicine for drug delivery and imaging: a promising avenue for cancer therapy and diagnosis using targeted functional nanoparticles. Int J Cancer. 2007;120:2527-2537.

4. Sharma P, Brown S, Walter G, Santa S, Moudgil B. Nanoparticles for bioimaging. Adv Colloid Interface Sci. 2006;123-126:471-485.

5. Doubrovin M, Serganova I, Mayer-Kuckuk P, Ponomarev V, Blasberg RG. Multimodality in vivo molecular-genetic imaging. Bioconjug Chem. 2004;15: 1376-1388.

6. Love Z, Wang F, Dennis J, et al. Imaging of mesenchymal stem cell transplant by bioluminescence and PET. J Nucl Med. 2007;48:2011-2020.

7. Wang W, Ke S, Kwon S, et al. A new optical and nuclear dual-labeled imaging agent targeting interleukin 11 receptor alpha-chain. Bioconjug Chem. 2007;18: 397-402.

8. Cai W, Chen X. Preparation of peptide-conjugated quantum dots for tumor vasculature-targeted imaging. Nat Protoc. 2008;3:89-96.

9. Chu TC, Shieh F, Lavery LA, et al. Labeling tumor cells with fluorescent nanocrystal-aptamer bioconjugates. Biosens Bioelectron. 2006;21:1859-1866.

10. Hicke BJ, Stephens AW, Gould T, et al. Tumor targeting by an aptamer. J Nucl Med. 2006;47:668-768.
11. Farokhzad OC. Quantum dot-aptamer conjugates for synchronous cancer imaging, therapy, and sensing of drug delivery based on bi-fluorescence resonance energy transfer. Nano Lett. 2007;7:3065-3070.

12. Girvan AC, Teng Y, Casson LK, et al. AGRO100 inhibits activation of nuclear factor- $\kappa \mathrm{B}(\mathrm{NF}-\kappa \mathrm{B})$ by forming a complex with NF- $\mathrm{B}$ essential modulator (NEMO) and nucleolin. Mol Cancer Ther. 2006;5:1790-1799.

13. Derenzini M, Sirri V, Trere D, Ochs RL. The quantity of nucleolar proteins nucleolin and protein B23 is related to cell doubling time in human cancer cells. Lab Invest. 1995;73:497-502.

14. Soundararajan S, Chen W, Spicer EK, Courtenay-Luck N, Fernandes DJ. The nucleolin targeting aptamer AS1411 destabilizes $\mathrm{Bcl}-2$ messenger RNA in human breast cancer cells. Cancer Res. 2008;68:2358-2365.

15. Ginisty H, Sicard H, Roger B, Bouvet P. Structure and functions of nucleolin. J Cell Sci. 1999;112:761-772.

16. Hovanessian AG, Puvion-Dutilleul F, Nisole S, Svab J, Perret E, Deng JS. The cell-surface expressed nucleolin is associated with the actin cytoskeleton. Exp Cell Res. 2000;261:312-328.

17. Yoon TJ, Yu KN, Kim E, et al. Specific targeting, cell sorting, and bioimaging with smart magnetic silica core-shell nanomaterials. Small. 2006;2:209-215.

18. So MK, Xu C, Loening AM, Gambhir SS, Rao J. Self-illuminating quantum dot conjugates for in vivo imaging. Nat Biotechnol. 2006;24:339-343.

19. Jeong JM, Hong MK, Chang YS, et al. Preparation of a promising angiogenesis PET imaging agent: ${ }^{68} \mathrm{Ga}$ labeled $\mathrm{c}(\mathrm{RGDyK})$-isothiocyanatobenzyl-1,4,7-triazacyclononane-1,4,7-triacetic acid and feasibility studies in mice. $\mathrm{J}$ Nucl Med. 2008;49:830-836.

20. Lee HY, Li Z, Chen K, et al. PET/MRI dual-modality tumor imaging using arginine-glycine-aspartic (RGD)-conjugated radiolabeled iron oxide nanoparticles. J Nucl Med. 2008;49:1371-1379.

21. Ray P, De A, Min JJ, Tsien RY, Gambhir SS. Imaging tri-fusion multimodality reporter gene expression in living subjects. Cancer Res. 2004;64: 1323-1330.

22. Antunes P, Ginj M, Zhang H, et al. Are radiogallium-labelled DOTA-conjugated somatostatin analogues superior to those labelled with other radiometals? Eur $J$ Nucl Med Mol Imaging. 2007;34:982-993.

23. Kim JS, Yoon TJ, Yu KN, et al. Toxicity and tissue distribution of magnetic nanoparticles in mice. Toxicol Sci. 2006;89:338-347. 